Recepción: 15 / 04 / 2019

Aceptación: 21 / 05 / 2019

Publicación: 05 / 07 / 2019

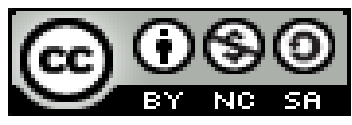

Ciencias económicas y empresariales

Artículo de investigación

\title{
El Presupuesto General del Estado y su aporte a la educación pública en el cantón
}

\section{Paján}

\section{The General State Budget and its contribution to public education in the Paján canton}

\section{Orçamento Geral do Estado e sua contribuição para a educação pública no cantão de Paján}

\author{
Gino Iván Ayón-Ponce ${ }^{\mathrm{I}}$ \\ gino.ayon@unesum.edu.ec \\ Carlos Artemidoro Zea-Barahona II \\ carlos.zea@unesum.edu.ec \\ Xavier Enrique Soledispa-Rodríguez ${ }^{\text {III }}$ \\ xavier.soledispa@unesum.edu.ec \\ Luís Alberto López-Párraga IV \\ luislopez@gmail.com
}

Correspondencia: gino.ayon@unesum.edu.ec

I. Magíster en Gerencia Educativa, Magíster en Administración de Empresas, Ingeniero Comercial, Profesor Titular Principal Carrera Administración de Empresas, Facultad de Ciencias Económicas Universidad Estatal del Sur de Manabí, Jipijapa, Manabí, Ecuador.

II. Magíster en Docencia Universitaria e Investigación Educativa, Economista, Profesor Titular Principal Carrera Administración de Empresas, Facultad de Ciencias Económicas de la Universidad Estatal del Sur de Manabí, Jipijapa, Manabí, Ecuador.

III. Especialista en Consultoría de Proyectos, Economista, Profesor Titular Principal Carrera Administración de Empresas, Facultad de Ciencias Económicas Universidad Estatal del Sur de Manabí, Jipijapa, Manabí, Ecuador. 


\title{
Resumen
}

La investigación realizada se fomentó en conocer "El presupuesto general del estado y su aporte a la educación pública en el cantón Paján, período 2015" cuyo objeto de estudio fue el presupuesto general del estado, mediante el objetivo general de analizar el porta a la educación pública en el cantón Paján, mediante un diagnóstico situacional reveló que el Distrito maneja recursos económicos, el monto asciende a la cantidad de USD 6'237,329.19, pero a su vez no son suficientes ya que la comunidad no ve reflejados los cambios como para mejorar todo el sistema educativo. La metodología desarrollada se basó en los métodos: científico, estadístico, descriptivo, inductivo, deductivo, analítico, conjuntamente con las técnicas de encuestas a los padres de familia del cantón Paján, con una muestra de 184 personas y entrevistas a los analistas del Distrito Educativo 13D09 Paján sirvieron como base para determinar conclusiones y recomendaciones.

Palabras clave: Proyectos; beneficiarios; políticas públicas; recategorización; capacitación.

\begin{abstract}
The research was encouraged to know "The general budget of the state and its contribution to public education in Paján canton, 2015" whose object of study was the general budget of the state, through the general objective of analyzing the portal to education public in the Paján canton, through a situational diagnosis revealed that the District manages economic resources, the amount amounts to the amount of USD 6'237,329.19, but at the same time they are not enough since the community does not see the changes reflected to improve everything the education system. The methodology developed was based on the following methods: scientific, statistical, descriptive, inductive, deductive, analytical, together with the survey techniques for Paján parents, with a sample of 184 people and interviews with the Educational District analysts. 13D09 Paján served as the basis for determining conclusions and recommendations.
\end{abstract}

Keywords: Projects; beneficiaries; public policies; recategorization; training.

\section{Resumo}

A pesquisa realizada foi incentivada a conhecer "O orçamento geral do estado e sua contribuição para a educação pública no cantão de Paján, período 2015" cujo objeto de estudo foi o orçamento geral do estado, através do objetivo geral de analisar o portal educacional. Público no cantão de Paján, através 
de um diagnóstico situacional revelou que o Distrito administra recursos econômicos, o montante equivale ao montante de US \$6.237.329,19, mas por sua vez não são suficientes já que a comunidade não vê as mudanças refletidas para melhorar tudo $\mathrm{O}$ sistema educativo A metodologia desenvolvida baseou-se nos métodos: científico, estatístico, descritivo, indutivo, dedutivo, analítico, juntamente com as técnicas de levantamento dos pais do cantão de Paján, com uma amostra de 184 pessoas e entrevistas com os analistas do Distrito Educacional. 13D09 Paján serviu de base para a determinação de conclusões e recomendações.

Palavras chaves: Projetos; beneficiários; políticas públicas; recategorização; treinamento.

\section{Introducción}

El presupuesto General del Estado es la estimación de los recursos financieros que tiene el Ecuador; es decir, aquí están los Ingresos (venta de petróleo, recaudación de impuestos, etc.) pero también están los Gastos (de servicio, producción y funcionamiento estatal para educación, salud, vivienda, agricultura, seguridad, transporte, electricidad, de acuerdo a las necesidades identificadas en los sectores y a la planificación de programas de desarrollo).

Las instituciones públicas harán una planificación priorizando sus necesidades para poder prestar sus bienes y servicios a la población. Por ejemplo, el Ministerio de Educación detalla las prioridades para atender a la población y brindar un buen servicio educativo. La educación es indispensable para el conocimiento, el ejercicio de los derechos y la construcción de un país soberano, y constituye un eje estratégico para el desarrollo nacional.

El presupuesto general del Estado, es la herramienta de gestión que tienen los gobernantes de turno para proyectar los ingresos y gastos públicos, esto les permite cumplir con la prestación de los bienes y servicios y cumplir sus políticas, planes, programas y proyectos que son indispensables para la satisfacción de las necesidades de la población.

Son muchos los sectores a los que está dirigido el presupuesto general del Estado en el Ecuador, dentro de estos sectores encontramos al educativo en todos sus niveles como lo son: el inicial, básico, bachillerato, técnico, superior y administración central, que entran dentro de las asignaciones que son entregadas por el Ministerio de Finanzas. 
La asignación presupuestaria cumple una serie de lineamientos, y deben cumplirse porque estas son normas establecidas en el marco legal que rigen las leyes ecuatorianas, en el campo educativo público es asignada de acuerdo a las necesidades que existen en cada uno de los distritos a nivel nacional. En muy importante hacer notar que el presupuesto del Ecuador se nutre de los ingresos petroleros y no petroleros, en estos últimos años el precio del petróleo ha disminuido, a tal medida que el presupuesto sufrió cambios, lo que afecta las asignaciones a todos los sectores económicos del país.

En el distrito educativo 13D09 del cantón Paján, mediante planificación operativa se evidencian las diferentes necesidades, entre las cuales se ven reflejadas falta de docentes para cubrir la planta óptima, el mantenimiento de la infraestructura educativa, implementación del programa dirigido a jóvenes y adultos con escolaridad inconclusa, el programa para jóvenes con rezago estudiantil, adquisición de materiales didácticos, de oficina, de aseo, mantenimiento de vehículos, de equipos informáticos. Los problemas que el gobierno central tiene debido a la disminución de los ingresos en el presupuesto general han causado que se originen estos problemas en los diferentes distritos del país.

\section{Presupuesto general del estado}

Para tener un concepto bien claro a que se refiere el presupuesto general del Estado (Dupuy, Julien, 2012) destaca lo siguiente:

Un presupuesto es la estimación de los ingresos (recursos) y gastos (desembolsos) para un periodo futuro. Al igual que una familia debe programar y ajustar sus gastos de acuerdo a los ingresos que percibe mensualmente, el Gobierno, realiza, cada año, la programación de los ingresos y gastos del Sector Público, el Presupuesto Público tiene tres grandes características, como es la estimación y programación de ingresos y gastos del Sector Público para una gestión. Es un instrumento de planificación y control que describe los planes y resultados esperados, expresados en la asignación de recursos; Por lo tanto, constituye la expresión financiera de los planes de gobierno. Es un instrumento de política fiscal, entendiéndose esta como parte de la política gubernamental que se refiere a la obtención de ingresos y las decisiones sobre el gasto público. 
El presupuesto general del Estado al ser un instrumento de planificación y control, al igual que una planificación familiar debe ajustarse de acuerdo a los ingresos que percibe, de acuerdo a esto se proyectan los gastos.

\section{Inversión Pública}

En lo correspondiente a las asignaciones de inversión pública, el (Ministerio de finanzas, 2014). Tienen como objetivo:

Fomentar y asegurar el desarrollo del país a largo plazo, especialmente con el apoyo a la transformación de la matriz productiva y energética, desarrollo del capital humano y protección social; toda vez que se considera la variable instrumental para lograr transformaciones estructurales que permitan tener una sociedad más justa, solidaria y, sobre todo, en la que pueda ejercer el buen vivir.

El ministerio de finanzas tiene como objetivo fomentar el desarrollo del País a largo plazo aportando de manera suficiente a la educación pública del Ecuador, tal y como lo específica en su PNBV 2015.

\section{Educación pública}

La educación según la (Constitución 2008) es indispensable para el conocimiento, el ejercicio de los derechos y la construcción de un país soberano, y constituye un eje estratégico para el desarrollo nacional destacando en sus artículos lo siguiente:

Art. 28.- La educación responderá al interés público y no estará al servicio de intereses individuales y corporativos. Se garantizará el acceso universal, permanencia, movilidad y egreso sin discriminación alguna y la obligatoriedad en el nivel inicial, básico y bachillerato o su equivalente. Es derecho de toda persona y comunidad interactuar entre culturas y participar en una sociedad que aprende. El Estado promoverá el diálogo intercultural en sus múltiples dimensiones.

La educación pública es el derecho que tenemos todos los ecuatorianos que sirve para la construcción de un país soberano, el cual debe brindarla de una manera gratuita y garantizando que sea laica sin importar raza, edad, género.

\section{El sistema educativo}

Según (definicionabc, s.f.), El sistema educativo es una creación del ser humano que tiene como objetivo principal permitir que: 
Una gran parte de la sociedad, si no toda, reciba el mismo tipo de educación y formación a lo largo de su vida. Se trata de la estructura general a través de la cual se organiza formalmente la enseñanza de una determinada nación. Este sistema que se ocupará de regir los destinos educacionales de un país siempre aparecerá manifestado de manera formal a través de una ley que expone explícitamente todos los componentes que intervienen en este proceso.

\section{La Educación en el Ecuador}

Según (Cantos, 2013) destaca lo siguiente sobre la educación en el Ecuador:

Está reglamentada por el Ministerio de Educación, dividida en educación fiscal, fisco misional, municipal, y particular; laica o religiosa, hispana o bilingüe intercultural. La educación pública es laica en todos sus niveles, obligatoria hasta el nivel básico, y gratuita hasta el bachillerato o su equivalente. Por otra parte la enseñanza, tiene dos regímenes, costa y sierra. Al régimen costa, pertenecen el litoral y las islas Galápagos, las clases comienzan a principios de abril de cada año y terminan en enero del siguiente año, en las vísperas de las vacaciones de invierno. Al régimen sierra la región interandina y Amazonía, inicia en septiembre de cada año y finalizan en junio del próximo año, justo a las vacaciones de verano, con una duración de casi 10 meses.

En Ecuador se estructura la educación pública de acuerdo a las condiciones climáticas, ya que solamente hay dos regímenes al año, en todos los dos sistemas es gratuita la educación, e inclusive de acuerdo a la Constitución de la República es gratuita hasta la educación superior.

\section{La Dirección Distrital 13D09 Paján - Educación: Presupuesto asignado}

En la Dirección Distrital 13D09 Paján - Educación desde el mes de enero a diciembre del año 2016, se asignó un presupuesto de \$5,377,253.00 con una modificación adicional de \$ 860,076.19 lo que resultó un presupuesto codificado de \$6,237,329.19 distribuido de la siguiente manera: En gastos de personal, denominado grupo 51, la cantidad de $\$ 5,863,595.67$; en Bienes y servicios de consumo, grupo 53, la cantidad de \$169,997.06; en Otros Gastos Corrientes, grupo 57, la cantidad de \$ 31,900.71; en Bienes y Servicios para Inversión, grupo 73, la cantidad de \$ 475.00; en Otros Pasivos, grupo 99, también denominado Deuda de años anteriores, la cantidad de \$16,105.79. 


\section{Materiales y Métodos}

Para el desarrollo del presente trabajo, se utilizaron los siguientes métodos de investigación: Científico, Estadístico Descriptivo Inductivo, Deductivo y Analítico con el apoyo de las técnicas de la observación, la encuesta y la entrevista. La población con la que contó fue de 354 padres de familias, de los cuales se tomó una muestra de 184 representantes.

\section{Resultados}

Entre los principales resultados se puede apreciar que el mayor rubro está en el grupo de gastos 51, o sea Gastos en Personal, lo que nos indica que el mayor gasto está en sueldos y salarios del personal docente, resumidos de la siguiente manera en tabla que a continuación se detalla:

Tabla 1: Presupuesto enero a diciembre del año 2016 Distrito 13D09 Paján

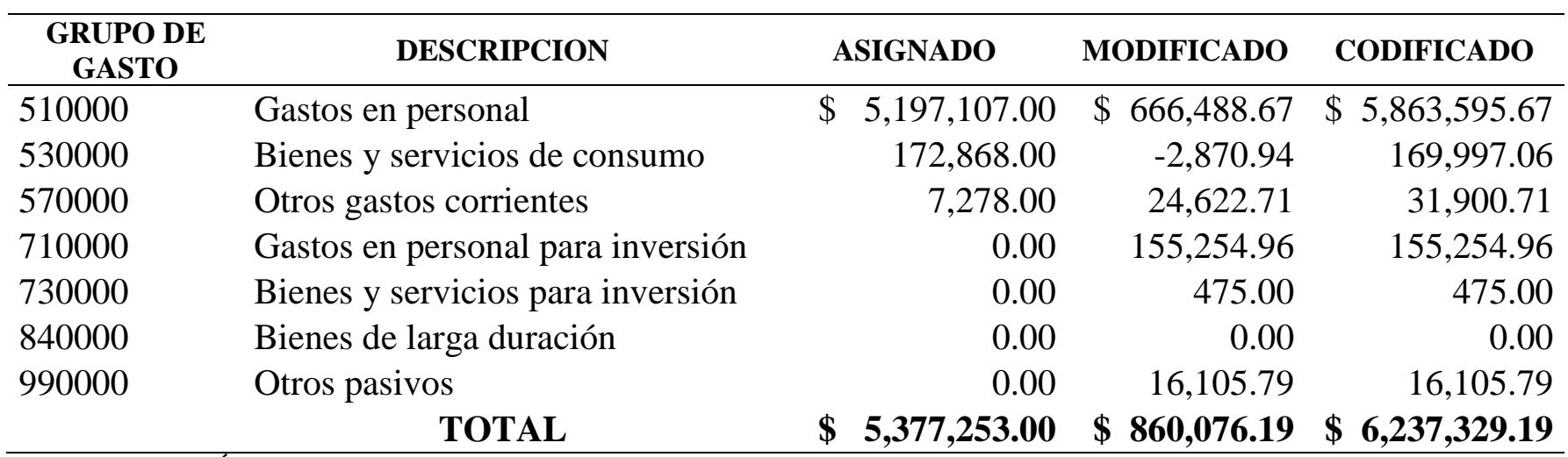

Fuente: Área financiera Dirección Distrital 13D09 Paján

Al preguntarle a los encuestados si dentro del presupuesto del distrito educativo 13D09 Paján existe un rubro destinado al mejoramiento de la infraestructura, como se puede apreciar en la tabla 2, entre definitivamente sí y probablemente sí suman el 64\%, lo que refleja el aporte del presupuesto general del Estado a la educación del cantón Paján:

Tabla 2: Rubro destinado al mejoramiento de la infraestructura

\begin{tabular}{ccc}
\hline Alternativa & Frecuencia & Porcentaje \\
\hline Definitivamente sí & $\mathbf{7 9}$ & $\mathbf{4 3 \%}$ \\
Probablemente sí & $\mathbf{3 8}$ & $\mathbf{2 1 \%}$ \\
Indeciso & $\mathbf{2 9}$ & $\mathbf{1 6 \%}$ \\
Probablemente no & $\mathbf{1 9}$ & $\mathbf{1 0 \%}$ \\
Definitivamente no & $\mathbf{1 9}$ & $\mathbf{1 0 \%}$ \\
Total & $\mathbf{1 8 4}$ & $\mathbf{1 0 0 \%}$ \\
\hline
\end{tabular}

Fuente: Padres de familias del sector educativo fiscal del cantón Paján 
Cuando se les consulto a los encuestados en razón de que se dieron mejoras en infraestructura y mobiliarios en las instituciones educativas y si esto ha permitido mejorar la educación en el cantón Paján, se evidencio con las respuestas que el27\% está muy de acuerdo en el aporte y el 39\% está de acuerdo, esto suma un total de 66\%, que saben el aporte que realizó el presupuesto general del Estado en el cantón Paján, tal como se lo muestra en la tabla 3:

Tabla 3: Mejoras en la educación en el cantón Paján

\begin{tabular}{ccc}
\hline Alternativa & Frecuencia & Porcentaje \\
\hline Muy de acuerdo & $\mathbf{5 0}$ & $\mathbf{2 7 \%}$ \\
De acuerdo & $\mathbf{7 1}$ & $\mathbf{3 9 \%}$ \\
Ni de acuerdo ni en desacuerdo & $\mathbf{4 6}$ & $\mathbf{2 5 \%}$ \\
En desacuerdo & $\mathbf{1 7}$ & $\mathbf{9 \%}$ \\
Total & $\mathbf{1 8 4}$ & $\mathbf{1 0 0 \%}$ \\
\hline
\end{tabular}

Fuente: Padres de familias del sector educativo fiscal del cantón Paján

\section{Discusión}

Se cita la investigación realizada por el Banco Mundial del autor (Torres, 1997) y titulada “¿Mejorar la calidad de la educación básica? Las estrategias del Banco Mundial":

La difusión de la educación ha reducido la pobreza al contribuir a que la economía de los países en desarrollo crezca a ritmos sin precedentes. Sin embargo, siguen habiendo todavía muchos problemas por resolver: ampliar el acceso en algunos países y, en muchos otros, aumentar la equidad, mejorar la calidad de la educación y acelerar el ritmo de la reforma educacional. Los sistemas actuales de financiamiento y de gestión no son con frecuencia adecuados para hacer frente a esos problemas. El gasto público en educación es muy a menudo ineficiente y poco equitativo. Dada la competencia por obtener fondos públicos y la presión sobre esos fondos, se necesitan nuevas fuentes de financiamiento. Además, es probable que se necesiten también cambios en la organización y la gestión de los sistemas de educación para permitir la flexibilidad y las posibilidades de elección que contribuyen a mejorar el rendimiento y las perspectivas de los estudiantes (BM, 1996:19).

Igualmente se cita la investigación de (Bonal, 2002) donde hace énfasis a los cambios de la educación en España: 
No hay duda de que el tipo de política educativa en España, a lo largo de los últimos veinte años, ha facilitado un acelerado proceso de transformación del sistema educativo, tanto en términos cuantitativos como cualitativos. Determinados indicadores, como el aumento de la demanda educativa en el sector público, la mejora relativa de las condiciones laborales del profesorado, el aumento del gasto público educativo, el incremento de las becas y ayudas al estudio o la cantidad de recursos materiales y humanos destinados a la reforma de la enseñanza secundaria, dan cuenta del impacto positivo de las políticas de igualdad y de calidad del sistema de enseñanza (Bonal, 1998).

También se consideró la investigación de (Becerra, España, \& Fiszbein, 2003) realizado en Argentina, donde se indicó:

En el marco del colapso dela economía Argentina ocurrido en 2002, el gasto público consolidado en educación sufrió la caída más importante de su historia disminuido un $38 \%$ en términos reales y un $28 \%$ en términos del Producto Bruto Interno (de un $4,7 \%$ a 3,4\%) respecto de 2001, aun cuando el PIB lo hizo en un $11 \%$ (...) Asimismo, cabe destacar que si bien en gasto público total consolidado cayó en un 39\% en 2002, la reducción del gasto en términos reales en educación ha sido mayor que las de las áreas de la salud y asistencia social.

Del mismo modo se hace referencia al estudio de (Díaz, 2007) respecto a la situación de la educación en el Ecuador donde se expresa:

El presupuesto destinado al sector educación crece en términos nominales de USD307,5 millones en el año 2000 a USD1.096,5 millones en el año 2006, sin que ello signifique mayor acceso y permanencia en la escuela ni mejora en la calidad. Con relación al gasto total del PGC y del PIB, el presupuesto del sector educación pasa del $7.1 \%$ al $12.8 \%$ y del $1.9 \%$ al $2.8 \%$ entre el 2000 y el 2006, respectivamente. Según la UNESCO, un "país debería gastar aproximadamente el 6\% de su PIB en educación para mantener un sistema eficiente y sostenible". 
En los últimos años, los gobiernos de los países en desarrollo han reconocido la importancia del presupuesto gubernamental. En la mayoría de ellos, el presupuesto ha desempeñado un papel puramente pasivo y las presiones sociales y políticas que se descargan sobre él han definido a la postre su estructura (...) En efecto, a medida que la intervención del Estado se fue haciendo necesaria para afronta fluctuaciones económicas que derivaban del exterior, fue considerándose que el presupuesto fiscal podrá ser utilizado como una eficaz herramienta de compensación frente a esas alteraciones (Martner, 2004).

\section{Conclusión}

En base a la investigación realizada se concluyó que un alto porcentaje de habitantes del cantón Paján, provincia de Manabí, si conoce que el Distrito Educativo maneja recursos económicos, pero a su vez no son suficientes ya que la comunidad no ve reflejados los cambios como para mejorar todo el sistema educativo.

Al detallar cuál es el rubro presupuestario sobre educación pública que ha sido asignado al distrito 13D09 del cantón Paján, se evidenció que el monto asciende a la cantidad de USD 6'237,329.19 (Seis millones doscientos treinta y siete mil trescientos veintinueve 19/100 dólares de los Estados Unidos de Norteamérica), pero su mayor porcentaje eta desina a pago de gastos en personal.

Al establecer de qué forma la ejecución presupuestaria destinada a infraestructura educativa influye en el mejoramiento del sistema educativo del cantón Paján, actualmente se concluye también que se ha mejora la infraestructura educativa y este si influye en estudiantes, padres y madres de familia, docentes y comunidad expresan que los espacios son más cómodos y agradables influyendo en el mejoramiento del sistema educativo del cantón Paján

\section{Referencias Bibliográficas}

Becerra, M., España, S., \& Fiszbein, A. (2003). Enfoques sobre la Eficiencia del Gasto en Educación Básica en la Argentina. Banco Mundial (Documento de Trabajo), 8. Recuperado el 23 de Julio de 2016 , de https://register.rti.org/EducationFinance/background/Accountability/argentina.pdf 
Bonal, X. (2002). El balance público-privado en el sistema de enseñanza español. . Educar, 27. $\begin{array}{llllll}\text { Recuperado el de } 23 \text { Julio de } & \text { 2016, }\end{array}$ http://ddd.uab.cat/pub/educar/0211819Xn29/0211819Xn29p11.pdf

Cantos, L. (15 de Enero de 2013). La educacion en el Ecuador. Recuperado el 22 de julio de 2016, de sistemaeducativoecuador.blogspot.com: http://sistemaeducativoecuador.blogspot.com/

Constitucion 2008. (s.f.). Recuperado el 22 de Julio de 2016, de www.asambleanacional.gob.ec: http://www.asambleanacional.gob.ec/sites/default/files/documents/old/constitucion_de_bolsil lo.pdf

definicionabc. (s.f.). Recuperado el 21 de Julio de 2016, de www.definicionabc.com: http://www.definicionabc.com/social/sistema-educativo.php

Díaz, G. V. (2007). Situación de la educación en el Ecuador. Educación, 16. Recuperado el 22 de Julio de 2016, de http://www.eumed.net/cursecon/ecolat/ec/2006/gfvd.pdf

Dupuy, Julien. (Febrero de 2012). Análisis del Presupuesto General del Estado 2012 - sector Salud Bolivia. Recuperado el 22 de julio de 2016, de https://books.google.com.ec/books?id=D3RLfFbLP3YC\&pg=PA15\&dq=PRESUPUESTO+ GENERAL+DEL+ESTADO\&hl=es-

419\&sa=X\&redir_esc=y\#v=onepage \&q=PRESUPUESTO\%20GENERAL\%20DEL\%20ES

TADO \& $\mathrm{f}=$ false

Martner, G. (2004). Planificación y presupuesto por programas. México: Siglo XXI. Recuperado el 20 de Julio $\quad$ de $2016, \quad$ de https://books.google.com.ec/books?id=UiedefaK9_4C\&pg=PA264\&dq=presupuesto+public o+concepto\&hl=es-

419\&sa=X\&redir_esc $=\mathrm{y} \# \mathrm{v}=$ onepage $\& \mathrm{q}=$ presupuesto $\% 20$ publico $\% 20$ concepto $\& \mathrm{f}=\mathrm{false}$

Torres, R. M. (1997). ¿Mejorar la calidad de la educación básica? Las estrategias del Banco Mundial. CORAGGIO, José Luis e TORRES, Rosa Maria. La educación segun el Banco Mundial: um análisis de sus propuestas y métodos. . Buenos Aires, Argentina: Nino y Darila Editores. $\begin{array}{lllllll}\text { Recuperado el de } & 22 \text { Julio de }\end{array}$ http://biblioteca.uahurtado.cl/ujah/reduc/pdf/pdf/8052.PDF 